\title{
Contralateral axillary metastasis: is surgical treatment the best option?
}

\author{
María Julia Giménez, Jesús Manuel Patrón, Giovanni Vento, Ana Bayón, Vincenzo Maisto, Isidro Bolumar, \\ Rosa Ferrer, Josep Asensi, Joaquin Gavila, Rafael Estevan \\ Department of Surgery, Fundación Instituto Valenciano de Oncología (IVO), Valencia 46007, España.
}

Correspondence to: Dr. María Julia Giménez, Department of Surgery, Fundación Instituto Valenciano de Oncología (IVO), Valencia 46007, España. E-mail: julia.gimenezcliment@gmail.com

How to cite this article: Giménez MJ, Patrón JM, Vento G, Bayón A, Maisto V, Bolumar I, Ferrer R, Asensi J, Gavila J, Estevan R. Contralateral axillary metastasis: is surgical treatment the best option? J Cancer Metastasis Treat 2019;5:28.

http://dx.doi.org/10.20517/2394-4722.2018.75

Received: 15 Nov 2018 First Decision: 13 Feb 2019 Revised: 20 Feb 2019 Accepted: 21 Feb 2019 Published: 8 Apr 2019

Science Editor: William Schiemann Copy Editor: Cai-Hong Wang Production Editor: Huan-Liang Wu

\begin{abstract}
Contralateral axillary metastasis (CAM) is a rare entity normally treated as a systemic disease. Recent publications have proposed aggressive surgical treatment with benefits to the patients in terms of survival. We present a case of a 74-year-old patient with a history of unilateral breast cancer, recurrence on the ipsilateral breast and then development of a CAM. The patient was treated with aggressive surgical treatment, but she developed an early recurrence of the disease with distant metastasis. There is limited evidence of the correct management of CAM, although proposed to treat it as a loco regional disease, individualized and multidisciplinary management is the best option for these patients.
\end{abstract}

Keywords: Breast cancer, contralateral axillary metastasis, lymphatic routes blockage

\section{INTRODUCTION}

Contralateral axillary metastasis (CAM), posterior to a unilateral breast cancer, is a rare entity. The reported incidence is between $1.9 \%$ and $6 \%$ in different publications ${ }^{[1,2]}$. Although rare, it is very clinically relevant, as lymph node metastasis is the most important prognostic factor in breast cancer. Correct identification of the primary origin of axillary metastasis is a priority; however, sometimes it can be a true challenge. There are many controversies about the pathophysiology and appropriate management of CAM because the contralateral axilla is considered a distant metastasis, thus stage IV disease, with limited therapeutic

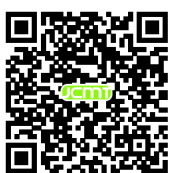




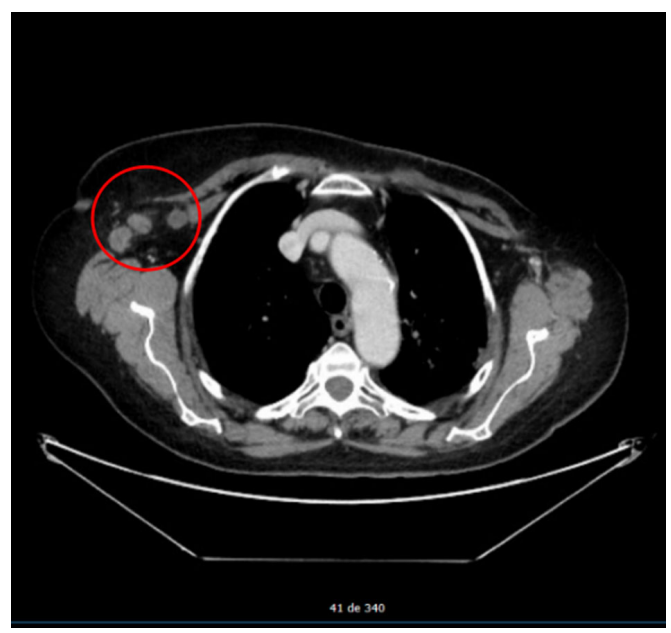

Figure 1. Computer tomography scan showing right axillary lymph node enlargement

strategies involved. Some authors have advised about a multidisciplinary and more aggressive approach to treat CAM, such as a loco regional spread breast cancer rather than distant metastasis ${ }^{[3,4]}$.

\section{CASE REPORT}

A 74-year old female, with an oncologic history of Hodgkin lymphoma (HL) stage IV B, with supra diaphragmatic and infra diaphragmatic adenopathies and bone marrow infiltration, was treated with chemotherapy twenty years ago.

The patient presented, in 2007, a left breast lesion on the upper outer quadrant (UOQ) detected by mammography. An ultrasound (US) and magnetic resonance imaging (MRI) study confirmed and classified the lesion as a BI-RADS 5. A core needle biopsy (CNB) was performed and the histopathology analysis (HA) reported an invasive ductal carcinoma (IDC), grade two, negative estrogen receptor (ER), positive progesterone receptor and negative human epidermal growth factor receptor type two (HER2/neu). A lumpectomy and sentinel lymph node biopsy (SLNB) with one step nucleic acid amplification (OSNA) technique, were performed. The SLN was negative and the size of the tumor was $1.5 \mathrm{~cm}$. Classified as a stage IA, complement treatment with radiotherapy (RT) and hormonal therapy with Anastrozole was decided for five years.

In 2016, a lesion of $2 \mathrm{~cm}$ in the surgical scar of the left breast was identified. Although the lesion was classified as an IDC, this time it was triple-negative (TN) by immunophenotype and with a Ki-67 of 35\%. A total left mastectomy and SLNB were indicated, with one positive lymph node in the OSNA analysis, thus requiring an axillary lymph node dissection (ALND). A total of 15 lymph nodes were excised without disease. Adjuvant systemic chemotherapy (ASC), with doxorubicin, cyclophosphamide, methotrexate and paclitaxel, was indicated.

The surgery was complicated with a chronic seroma, and it was managed with conservative measures for six months without success; thus, requiring surgical treatment. During the procedure, in August 2017, a suspicious lesion was observed in the surgical bed of the mastectomy. A biopsy was taken, reporting metastatic infiltration of carcinoma in the pectoralis major. A wide local excision of the pectoralis major muscle was performed and the HA reported an IDC of $3.5 \mathrm{~cm}$, grade three, TN, Ki67 of 75\% and free margins. With this result, the patient underwent ASC with Capecitabine plus RT.

In March 2018, suspicious right axillary lymph nodes were identified during a computer tomography scan [Figure 1]. The study, complemented with US and MRI, identified an intramammary lymph node 


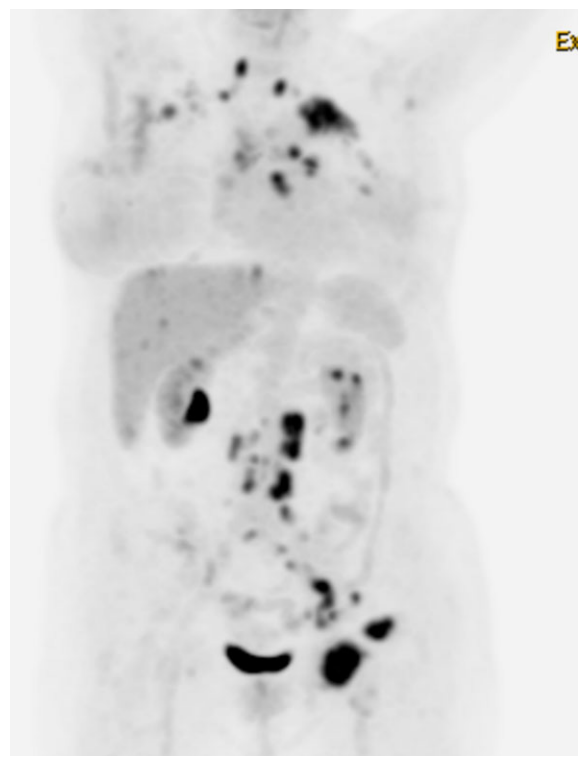

Figure 2. Positron emission tomography computer tomography showing multiple metastatic disease

in the UOQ of the right breast. Surgical treatment was determined and 43 out of 45 of the right ALND were positive, seven of them with capsular rupture. In addition, the intramammary lymph node was also positive for IDC, TN immunophenotype with a Ki67 of 63\%. ASC and RT to the whole breast, axillary and supraclavicular region was decided.

During controls, in May 2018 the patient complained of a lump in the left inguinal area, when performing the physical exploration, a three $\mathrm{cm}$ lymph node was identified. A CNB reported metastatic carcinoma compatible with breast cancer. A positron emission tomography (PET) scan revealed multiple abnormal lymph nodes in the cervical, supraclavicular, mediastinal, retroperitoneal and inguinal regions. In addition, two bone lesions, one in the pelvis and another in the humeral bone were described as well as unspecific pleural nodules [Figure 2].

Treatment with carboplatin and gemcitabine was administered. However, a new PET revealed disease progression with new muscular, hepatic, subcutaneous and bone lesions. The patient was admitted to the hospital for severe respiratory insufficiency due to bilateral pleural effusion; finally, she died on October 10th of 2018.

\section{DISCUSSION}

One of the main explanations of CAM is aberrant drainage due to blockage of normal lymphatic routes. Perre et al. ${ }^{[5]}$ described this theory; they studied 23 patients with breast cancer who underwent lymphoscintigraphy before and after surgical treatment with or without radiotherapy. Only one patient presented drainage to the contralateral axilla before treatment; however, this same event presented in four patients after ALND of the opposite axilla and in one patient after radiotherapy. In a more recent publication, van der Ploeg et al. ${ }^{[6]}$ observed, in patients with previous ALND for breast carcinoma, contralateral axillary drainage in four out of 12 of them, during lymphoscintigraphy and SLNB. This finding becomes more relevant because two of the four involved lymph nodes were positive for metastasis. Both patients underwent ALND on the same side and reported alive and free of disease for 22 and 36 months after the procedure. In these two publications, the authors concluded that this mechanism of drainage reflects regional dissemination to the node on the direct pathway, making the "crossover metastasis" effect. However, a different outcome would be in cases of untreated breast cancer, where CAM means dissemination beyond the regional nodes. 
Other risk factors for CAM have been described, including obstruction of the ipsilateral lymphatic drainage, bulky tumor in the breast, extensive tumor burden in the axilla, and as mentioned before, previous surgery and/or radiotherapy ${ }^{[3,7]}$.

There are challenges in the clinical scenario of CAM. Contralateral spread from the original tumor is not the only possibility. An occult second primary in the contralateral breast and axillary metastasis from the extrammamary site should be considered ${ }^{[8]}$. Although it is possible to have certainty of the tumor origin with a biopsy of the axillary node, to discard an occult second primary in the contralateral breast is not always an easy task. MRI has been proposed to be a good technique to identify occult primary breast carcinoma (OPBC). In a prospective analysis carried out by Buchanan et al $.^{[9]}, 69$ patients with occult OPBC, underwent breast MRI, identifying $80 \%$ (55 of 69 ) of suspicious lesions and proved to be breast carcinoma in $49 \%$ of them ( 34 of 69 ). Although MRI has its limitations, due to false positive results, the authors of this study support that OPBC is a primary indication for the use of MRI.

CAM is an uncommon event in breast cancer and has a poor prognosis. AJCC 8th edition classifies CAM as stage IV disease because contralateral axillary lymph nodes are not considered a regional extent of the disease, but a distant metastasis ${ }^{[10]}$. However, several publications have questioned this affirmation and proposed that treatment of CAM should be aggressive and multidisciplinary as it is in the case of locally advanced breast cancer, rather than treated as a distant metastasis ${ }^{[3,4,8]}$. A systematic review analyzed 48 patients with CAM, 23 had complete follow up data during a mean time of 50.3 months with an overall survival of $82.6 \%$. An important fact is that $92.1 \%$ of patients received surgical treatment and $88.9 \%$ systemic treatment ${ }^{[11]}$.

The patient of the case had many risks factors, like conservative surgery, radiotherapy in the left breast and an ipsilateral recurrence treated with mastectomy and SLNB. After completing ALND, 15 lymph nodes were negative for metastasis. However, CAM was identified two years later.

History of HL has been described as a risk factor for breast cancer, with an excess risk of $40 \%$ even after 40 years. The main risk factor is mantle radiotherapy, especially in young women in the "peri-pubertal" period, corresponding as the highest breast radio sensitivity period ${ }^{[12,13]}$. The patient in our case was treated with chemotherapy exclusively and after menopause, thus we cannot assume a specific risk association.

It is important to mention that even though this patient received aggressive surgical treatment, chemotherapy and radiotherapy, the disease progressed as a systemic disease in a short period of time. This reflects the complexity of the case and that different approaches should be taken into account when treating these patients.

In conclusion, CAM are an unusual form of metastasis in unilateral breast cancers. The assumption of a disseminated disease prompt to systemic therapies rather than an aggressive surgical treatment. There are many controversies regarding the appropriate management and the prognostic influence on these patients. Unfortunately, there is poor evidence for the adequate treatment, due to the rarity of the event. An individualized and multidisciplinary approach is encouraged for these patients, so a positive impact on survival is achieved.

\section{DECLARATIONS}

\section{Authours' contributions}

Conception or design of the work: Giménez MJ, Patrón JM

Data collection: Giménez MJ, Patrón JM, Vento G, Ferrer R, Asensi J, Gavila J

Drafting, critical revision of the article, final approval of the version to be published: Giménez MJ, Patrón

JM, Vento G, Bayón A, Maisto V, Bolumar I, Ferrer R, Asensi J, Gavila J, Estevan R 


\section{Avalibility of data and materials}

Not applicable.

\section{Financial support and sponsorship}

None.

\section{Conflicts of interest}

The authors declared that there are no conflicts of interest.

\section{Ethical approval and consent to participate}

Not applicable.

\section{Consent for publication}

Not applicable.

\section{Copyright}

(c) The Author(s) 2019.

\section{REFERENCES}

1. Huston T, Pressman P, Moore A. The presentation of contralateral axillary lymph node metastases from breast carcinoma: a clinical management dilemma. Breast J 2007;13:158-64.

2. Morcos B, Jaradat I, El-Ghanem M, Characteristics of and therapeutic options for contralateral axillary lymph node metastasis in breast cancer. EJSO 2011;37:418-21.

3. Chkheidze R, Sanders M.A, Haley B. Isolated contralateral axillary lymph node involvement in breast cancer represents a locally advanced disease not distant metastases. Clin Breast Cancer 2017.

4. Kiluk J, Prowler V, Lee M.C. Contralateral axillary nodal involvement from invasive breast cancer. Breast J 2014;23:291-4.

5. Perre CI, Hoefnagel CA, Kroon BB. Altered lymphatic drainage after lymphadenectomy or radiotherapy of the axilla in patients with breast cancer. Br. J. Surg 1996;83:1258.

6. van der Ploeg I, Oldenburg H, Rutgers E. Lymphatic drainage patterns from the treated breast. Ann Surg Oncol. 2010;17:1069-75.

7. Allweis T, Parson B, Klein M. Breast cancer draining to bilateral axillary lymph nodes. Surgery 2003;134:506-8.

8. Wang W, Yuan P, Wang J, et al. Management of contralateral axillary lymph node metastasis from breast cancer: a clinical dilemma. Tumori 2014;100:600-4.

9. Buchanan C, Morris E, Dorn P. Utility of breast magnetic resonance imaging in patients with occult primary breast cancer. Ann Surg Oncol 2005;12:1045-53.

10. Amin MB, Edge S. AJCC cancer staging manual. 8th ed. Springer; 2017.

11. Moossdorff M, Vugts G, Maaskant-Braat AJ, Strobbe LJ, Voogd AC, et al. Contralateral lymph node recurrence in breast cancer: regional event rather than distant metastatic disease. A systematic review of the literature. Eur J Surg Oncol 2015;41:1128-36.

12. Cutuli B, Kanoun S, Tunon De Lara C. Breast cancer occurred after Hodgkin's disease: Clinico-pathological features, treatments and outcome: analysis of 214 cases. Crit Rev Oncol Hematol 2012;81:29-37.

13. Shaapveld M, Aleman B, van Eggermond A. Second cancer risk up to 40 years after treatment for Hodgkin's lymphoma. N Engl J Med 2015;373:2499-511. 